\title{
Comportamento sedentário como critério discriminador do excesso de peso corporal em idosos
}

\author{
Sedentary behavior as a discriminating criterion for overweight in the elderly
}

\section{AUTORES \\ Andréia dos Santos Jesus ${ }^{1}$ (D) \\ Saulo Vasconcelos Rocha ${ }^{1}$ (iD \\ 1 Universidade Estadual do Sudoeste da Bahia, \\ Departamento de Saúde II, Jequié, Bahia, Brasil.}

\section{CONTATO}

Saulo Vasconcelos Rocha

svrocha@uesb.edu.br

Avenida José Moreira Sobrinho S/N, Jequiezinho, Jequié, Bahia, Brasil.

CEP: 45206-190.

DOI

$10.12820 /$ rbafs. $23 \mathrm{e} 0030$

\begin{abstract}
RESUMO
O objetivo do estudo foi analisar o comportamento sedentário como discriminador de excesso de peso corporal em idosos. Estudo transversal, com 310 idosos (idade $\geq 60$ anos), de ambos os sexos, residentes no município de Ibicuí, Bahia. Foram investigadas informações sobre características sociodemográficas e pessoais; hábitos de vida e comportamento sedentário (CS), avaliado por meio do Questionário Internacional de Atividade Física - IPAQ. O índice de massa corporal (IMC) foi calculado pela divisão da massa corporal $(\mathrm{kg})$ pelo quadrado da estatura $\left(\mathrm{m}^{2}\right)$. Para identificação do CS (total/dia) como discriminador de excesso de peso, foi adotada análise das curvas Receiver Operating Characteristic (ROC) com IC95\%. Os resultados apontaram tempo médio de CS de 414,14 ( \pm $243,80) \mathrm{min} /$ dia e a prevalência de excesso de peso $\left(\mathrm{IMC}>27 \mathrm{~kg} / \mathrm{m}^{2}\right.$ ) foi de $39,7 \%$. A área total sob a curva ROC entre CS e o excesso de peso foi 0,59 (IC95\%: 0,50-0,67) para homens e 0,54 (IC95\%: 0,43-0,58) para mulheres. Os melhores pontos de corte para discriminar a presença de excesso de peso foram de $390 \mathrm{~min} /$ dia ou mais e $270 \mathrm{~min} /$ dia ou mais para homens e mulheres, respectivamente. Nesse sentido, observa-se que o tempo gasto em CS apresenta uma boa capacidade preditiva para discriminar o excesso de peso em idosos.
\end{abstract}

Palavras-chave: Estilo de vida sedentário; Obesidade; Envelhecimento; Idoso.

\section{ABSTRACT}

The objective of this study was to analyze sedentary behavior as a discriminator of overweight in the elderly. A cross-sectional study with a sample of 310 individuals (age $\geq 60$ years) of both sexes living in the municipality of Ibicui, Bahia, Brazil. Information on sociodemographic and personal characteristics was investigated; life habits and sedentary behavior (assessed through the International Physical Activity Questionnaire - IPAQ). The body mass index (BMI) was calculated by dividing body mass (kg) by the square of height (m2). In the data analysis, descriptive statistics procedures were used. In order to identify $S B$ as an excess discriminator, we adopted an analysis of the Receiver Operating Characteristic (ROC) curves with $95 \%$ CI. The results of the study showed that mean SB time was 414.14 ( 243.80) $\mathrm{min} /$ day and the prevalence of overweight $\left(B M I>27 \mathrm{~kg} / \mathrm{m}^{2}\right)$ was $39.7 \%$. The total area under the ROC curve between SB and overweight was 0.59 (95\%CI: 0.50-0.67) for males and 0.54 (95\% CI: 0.43-0.58) for women. The best cutoff points to discriminate the presence of excess weight were 390 $\mathrm{min} /$ day or more and $270 \mathrm{~min} /$ day or more for men and women, respectively. Thus, it is observed that the time spent in SB has a good predictive capacity to discriminate overweight in the elderly.

Keywords: Sedentary lifestyle; Obesity; Aging; Aged.

\section{Introdução}

O comportamento sedentário (CS) pode ser compreendido como tempo gasto em atividades realizadas em posições deitadas, reclinadas, sentadas, que não tenham gasto energético acima dos valores de repouso $(<1,5$ METs $)^{1}$. Dentre as atividades sedentárias, a exemplo, dirigir carro, assistir televisão, assistir aulas, utilização de computador, smartphone, entre muitas outras atividades do cotidiano, podendo ocorrer em diferentes ambientes: casa, faculdade, trabalho, deslocamento e lazer ${ }^{2,3}$.

O CS tem sido apontado como um potencial fator de risco para doenças crônicas (diabetes tipo 2, doença cardiovascular e câncer $)^{4,5}$ e mortalidade por todas as causas $^{8}$. Esse risco pode ser atenuado (mais não eliminado) pelo acúmulo de atividades físicas moderadas/ intensas (60 a 75 minutos por dia) ${ }^{7}$.

O tempo despendido em atividades sedentárias é um tipo de comportamento que está cada vez mais presente entre pessoas de diferentes faixas etárias ${ }^{8,9}$. Especificamente, entre a população idosa, é consenso que com avançar dos anos, os níveis de gasto energético diário declinam e ocorre um incremento do tempo em $\mathrm{CS}^{10}$.

Por outro lado, o excesso de peso é uma doença crônica metabólica, considerada um problema de saúde 
pública em vários países ${ }^{11,12}$, com elevada prevalência entre idosos brasileiros. Um estudo de abrangência nacional mostrou que a prevalência geral de excesso de peso em idosos brasileiros foi de $45,2 \%$ sendo mais acentuado na região sul do país ${ }^{13}$.

O excesso de peso caracteriza-se por acúmulo de tecido adiposo no organismo e sua origem está associada a fatores metabólicos, genéticos, hábitos nutricionais (hipercalóricos), falta de atividade física e idade (redução de massa corporal e gasto energético) ${ }^{14}$. Essa condição provoca alteração no perfil metabólico, reduz a tolerância à glicose, eleva a resistência à insulina e os perfis lipídicos ${ }^{14}$, aumentando o risco de doenças crônicas não transmissíveis (DCNTs) e mortalidade ${ }^{11,15}$. Entre a população de idosos, o excesso de peso repercute em um declínio da qualidade de vida ${ }^{16}$ e limitações no desempenho das atividades diárias ${ }^{17,18}$.

Achados da literatura demonstraram que elevados volumes de tempo despendido em atividades sedentárias estão relacionados a incrementos no risco de obesidade $^{4,5}$. No entanto, o conhecimento sobre a relação entre o tempo exposto ao CS e o excesso de peso na população idosa ainda são incipientes, como demonstrados por Santos et al. ${ }^{19}$ em estudo de revisão sistemática. Os autores apontaram que existe uma indefinição sobre qual a quantidade de tempo em atividades sedentárias representa risco a saúde de indivíduos idosos. Diante do exposto, o propósito deste estudo é analisar o comportamento sedentário como discriminador de excesso de peso em idosos.

\section{Método}

Este estudo foi aprovado pelo Comitê de Ética em Pesquisa da Universidade Estadual do Sudoeste da Bahia (Parecer $n^{\circ}$ 613.364/2014) seguindo os princípios éticos da resolução no 466/2012 do Conselho Nacional de Saúde (CNS).

Trata-se de um estudo epidemiológico de corte transversal. Os dados foram extraídos do inquérito domiciliar intitulado - Monitoramento das Condições de Saúde de Idosos de um Município de Pequeno Porte (MONIDI), realizado com idosos no município de Ibicuí, Bahia, Brasil, no ano de 2014.

Ibicuí está localizada na região sudoeste do estado baiano, possui uma área territorial de $1.176,843 \mathrm{Km}^{2}$, a população de 2013 de 15.785 habitantes, sendo 2.125 idosos, dos quais 525 estavam cadastrados nas Unidades de Saúde da Família (USF) durante o período da coleta de dados.

A população alvo do presente estudo foi constituída por indivíduos com idade igual ou superior a sessenta anos, cadastrados pelas USF do município. Foram excluídos todos os idosos acamados, com diagnóstico de Alzheimer ou outro tipo de doença neurológica (com diagnóstico registrado em prontuário médico da Unidade de Saúde da Família) que comprometesse a veracidade das informações.

Para a determinação do tamanho da amostra foram baseados nos critérios propostos por Luiz \& Magna$\mathrm{ni}^{20}$ para populações finitas. A amostra foi obtida assumindo a prevalência estimada do evento de interesse como desconhecida (50,0\%) erro amostral de 3,0\% e $95,0 \%$ de confiança.

Os participantes foram selecionados de forma aleatória através de sorteio dos idosos cadastrados na USF, sendo este proporcional ao tamanho e a distribuição por sexo e por USF. A amostra final do estudo foi constituída por 310 idosos (201 zona urbana e 109 zona rural) - Figura 1.

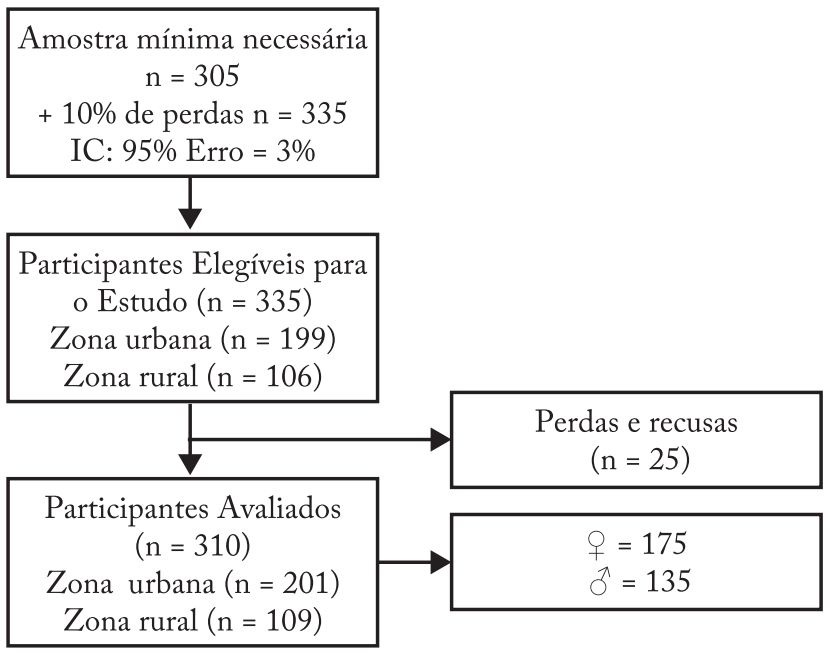

Figura 1 - Organograma de distribuição da amostra do estudo Monitoramento das Condições de Saúde de Idosos de um Município de Pequeno Porte (MONIDI). Ibicuí, Brasil, 2014.

Os dados foram coletados por meio de um questionário denominado Instrumento de Avaliação da Saúde de Idosos (IASI) padronizado e validado previamen$\mathrm{te}^{21}$, aplicado em forma de entrevista individual, seguido de avaliação antropométrica.

No presente estudo foram incluídas as seguintes variáveis: sociodemográficas: sexo (masculino/feminino), idade: em anos completos e categorizada por faixa etária (60 - 79 anos/80 anos ou mais); situação conjugal atual: (com companheiro/sem companheiro); escolaridade: (alfabetizado/não alfabetizado); renda mensal familiar em reais; hábitos de vida: fuma atualmente ou já 
foi fumante ( $\operatorname{sim} /$ não), consumo de bebidas alcoólicas ( $\operatorname{sim} /$ não), consumo de verduras, legumes e frutas ( $\operatorname{sim} /$ não); comportamento sedentário: avaliado por meio de questões retiradas do Questionário Internacional de Atividade Física (IPAQ) versão adaptada para idosos brasileiros $^{22}$ que permite mensurar o tempo sentado durante um dia da semana e um dia do final de semana (horas/minutos). O tempo gasto em CS foi registrado em minutos e o escore final foi obtido a partir do cálculo do tempo gasto sentado $(\mathrm{min} / \mathrm{semana})=$ minutos $\mathrm{em}$ posição sentada em um dia de semana * 5 (cinco) dias úteis + minutos sentados em um dia do final de semana * 2 (dois). Para verificar o tempo médio, o valor obtido foi dividido por sete (equivalente ao número de dias em uma semana); Inatividade Física no Lazer: sim (não participa de no mínimo 150 minutos por semana de atividade física moderada/vigorosa por semana no seu tempo livre), não (participa de no mínimo 150 minutos de atividade física moderada/vigorosa por semana no tempo livre).

As medidas antropométricas foram mensuradas (massa corporal e estatura) e avaliada por profissional previamente treinado utilizando métodos padroniza$\operatorname{dos}^{23}$. O índice de massa corporal (IMC) foi calculado através da divisão do peso $(\mathrm{kg})$ pela estatura elevada ao quadrado $\left(\mathrm{m}^{2}\right)$. Os pontos de corte para classificação do excesso de peso foi de $\geq 27 \mathrm{Kg} / \mathrm{m} 2^{24}$.

Analisou-se os dados pelo sofware Statistical Package for the Social Scienses versão 22.0 e Medcalc ${ }^{\circledR}$ versão 17.9. $\mathrm{Na}$ análise descritiva, calculou-se os valores de média e desvio padrão ou frequência absoluta e relativa e medidas de associação para variáveis categóricas (Teste Qui-quadrado) com $\mathrm{p}<0,05$.

A capacidade discriminatória, sensibilidade e especificidade do CS para avaliar a presença de excesso de peso foram estabelecidas a partir da análise das curvas Receiver Operating Characteristic (ROC). A área sob a curva ROC (ASC) determina a capacidade discriminatória do indicador para presença ou ausência de excesso de peso.

\section{Resultados}

A média de idade dos entrevistados foi de 71,62 $( \pm 8,15)$ anos, com predominância do sexo feminino $(58,1 \%)$. A renda média mensal foi de 708,26 \pm $303,69)$ reais. O tempo médio despendido em comportamento sedentário (CS) foi de 414,14 ( $\pm 243,80) \mathrm{min} /$ dia $(437,05 \pm 241,28 \mathrm{~min} /$ dia para os homens e 396,36 $\pm 244,96 \mathrm{~min} /$ dia para as mulheres). As prevalências de inatividade física no lazer e de excesso de peso foram de $58,9 \%$ (65,2\% entre os homens e $72,0 \%$ entre as mulheres) e $39,7 \%$ (42,5\% entre os homens e $37,3 \%$ entre as mulheres), respectivamente. Foi identificada associação entre sexo e a variável situação conjugal ( $\mathrm{p}<$ $0,05)$, com maior percentual de mulheres vivendo sem companheiro $(63,9 \%)$ - Tabela 1.

Tabela 1 - Características sociodemográficas, comportamentais, antropométricas dos idosos residentes do município de Ibicuí, Bahia, Brasil, 2014.

\begin{tabular}{|c|c|c|c|c|c|}
\hline \multirow{3}{*}{ Variáveis } & \multicolumn{4}{|c|}{ Sexo } & \multirow{3}{*}{$\mathrm{p}$} \\
\hline & \multicolumn{2}{|c|}{$\begin{array}{c}\text { Masculino } \\
(\mathrm{n}=135)\end{array}$} & \multicolumn{2}{|c|}{$\begin{array}{l}\text { Feminino } \\
(\mathrm{n}=175)\end{array}$} & \\
\hline & $\mathrm{n}$ & $\%$ & $\mathrm{n}$ & $\%$ & \\
\hline Idade & & & & & 0,188 \\
\hline 60 a 79 anos & 109 & 41,9 & 151 & 58,1 & \\
\hline 80 anos ou mais & 26 & 52,0 & 24 & 48,0 & \\
\hline \multicolumn{6}{|l|}{ Situação conjugal } \\
\hline Com companheiro & 78 & 51,3 & 74 & 48,7 & $0,007^{*}$ \\
\hline Sem companheiro & 57 & 36,1 & 101 & 63,9 & \\
\hline \multicolumn{6}{|l|}{ Escolaridade } \\
\hline Alfabetizado & 76 & 43,7 & 98 & 56,3 & 0,958 \\
\hline Não-alfabetizado & 59 & 43,4 & 77 & 56,6 & \\
\hline \multicolumn{6}{|c|}{ Consumo de frutas, verduras legumes } \\
\hline Sim & 7 & 46,7 & 8 & 53,3 & 0,803 \\
\hline Não & 128 & 43,4 & 167 & 56,6 & \\
\hline \multicolumn{6}{|l|}{ Álcool } \\
\hline Sim & 9 & 69,2 & 4 & 30,8 & 0,058 \\
\hline Não & 126 & 42,6 & 170 & 57,4 & \\
\hline \multicolumn{6}{|l|}{ Fumo } \\
\hline Sim & 21 & 58,3 & 15 & 41,7 & 0,057 \\
\hline Não & 114 & 41,6 & 160 & 58,4 & \\
\hline \multicolumn{6}{|l|}{ Inatividade física no lazer } \\
\hline Não (< 149 min/semana) & 47 & 49,0 & 49 & 51,0 & 0,198 \\
\hline $\operatorname{Sim}(\geq 150 \mathrm{~min} / \mathrm{semana})$ & 88 & 41,1 & 126 & 58,9 & \\
\hline \multicolumn{6}{|l|}{ Excesso de peso } \\
\hline Sim & 57 & 47,9 & 62 & 52,1 & 0,361 \\
\hline Não & 77 & 42,5 & 104 & 57,5 & \\
\hline
\end{tabular}

*p $>0,005$

Após a construção das curvas ROC, identificou-se que o CS apresentou Área sob a Curva (ASC) com escores de 0,59 (IC95\%: 0,50-0,67) para os homens (Figura 2) e 0,54 (IC95\%: 0,43-0,58) para as mulheres (Figura 3).

Ao analisar os pontos de corte com maior acurácia e valores de sensibilidade e especificidade da relação entre o CS e o excesso de peso, observou-se que entre as mulheres o ponto de corte no CS que discriminou o excesso de peso foi de mais de $270 \mathrm{~min} / \mathrm{dia}$ (sensibilidade $=58,1 \% \mathrm{e}$ 
especificidade $=44,7 \%$ ) e entre os homens de mais de 390 $\mathrm{min} /$ dia $($ sensibilidade $=50,9 \%$ e especificidade $=58,4$ ).

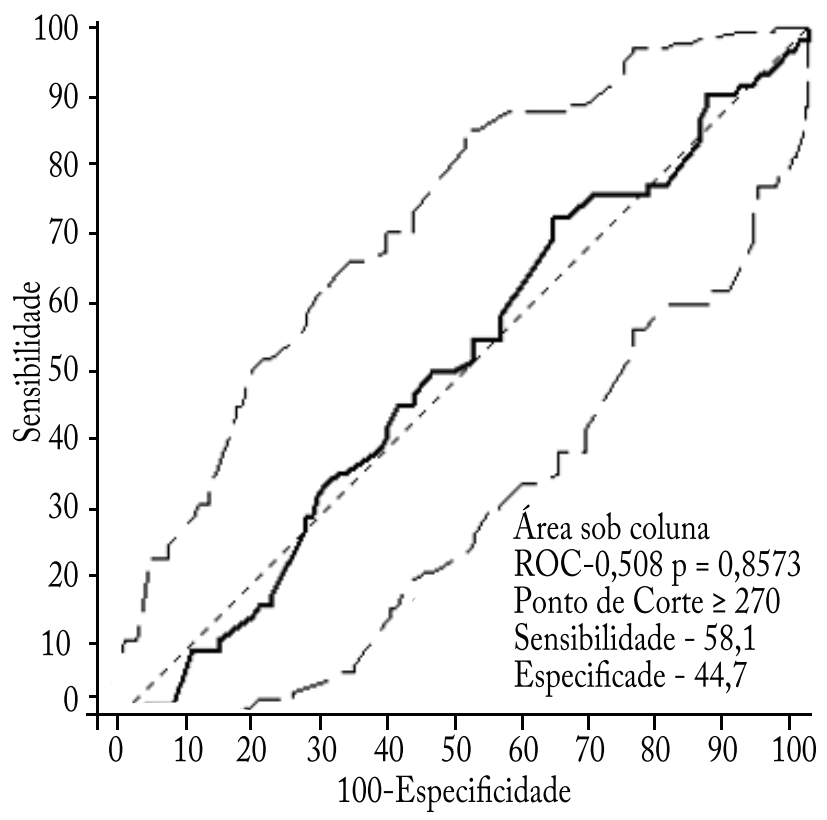

Figura 2 - Área sob a curva (ROC) com ponto de corte para o CS como discriminador do excesso de peso em mulheres idosas residentes em Ibicuí, Bahia, Brasil, 2014 ( $\mathrm{n}=310)$.

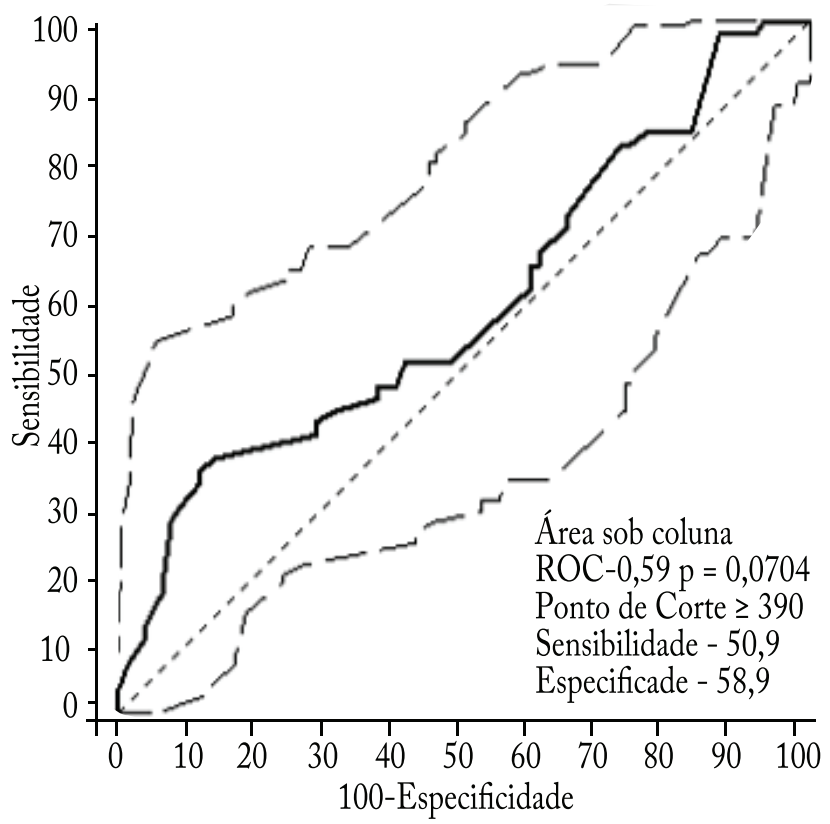

Figura 3 - Área sob a curva (ROC) com ponto de corte para o CS como discriminador do excesso de peso em homens idosos residentes em Ibicuí, Bahia, Brasil, $2014(\mathrm{n}=310)$.

\section{Discussão}

Este estudo teve como objetivo analisar o comportamento sedentário como discriminador de excesso de peso em idosos. Nossos resultados demonstraram que o CS apresenta uma capacidade discriminatória para dis- criminar o excesso de peso diferente entre os sexos, com maior potencial entre os homens. Os tempos despendidos em atividades sedentárias que melhor discriminaram o excesso de peso foram de $270 \mathrm{~min} / \mathrm{dia}$ ou mais para mulheres e $390 \mathrm{~min} /$ dia ou mais para os homens.

Compreende-se que dentre as mudanças associadas ao processo de envelhecimento, as mudanças na composição corporal são frequentes com aumentos progressivos na deposição e distribuição da gordura corporal, com redução da gordura na região dos membros e um elevado acúmulo na região abdomina ${ }^{25}$.

Os mecanismos fisiológicos para justificar os efeitos adversos à saúde decorrentes dos elevados tempos despendidos em CS estão associados a maior liberação de macrófagos ativos ${ }^{26}$. Essas células têm a função de produzir citocinas pró-inflamatórias responsáveis por desempenhar um papel fundamental na patogênese das doenças metabólicas e cardiovasculares ${ }^{26}$.

Além disso, a imobilização muscular proporcionada pela supressão da contração muscular favorece a diminuição da atividade enzimática lipoproteína lipase (LPL), ocasionando uma redução na absorção de triglicérides, e menor produção de HDL-c e glicose plasmática. Com o decréscimo da atividade enzimática da LPL, a concentração de HDL no sangue também diminui, tendo como consequência o aumento da exposição para doenças cardiovasculares. Assim, a redução do tempo em CS, contribui para uma maior ativação da função da enzima LPL, reduzindo os efeitos prejudiciais ao metabolismo dos lipídios na produção de $\mathrm{HDL}^{27}$.

Desse modo, os idosos, devem ser estimulados a reduzir o tempo de exposição ao CS, com o propósito de controlar o processo de desenvolvimento e/ou piora das doenças crônicas não transmissíveis, cardiovasculares e consequentemente, favorecer o processo de envelhecimento saudável.

Resultados de estudos realizados com idosos de diferentes países como Estados Unidos ${ }^{28}$, Espanha ${ }^{29}$ e Brasi $^{30}$ mostraram uma associação direta entre CS e excesso de peso. Entretanto, aspectos como a idade dos participantes, bem como os diferentes pontos de corte do IMC utilizados para classificação do excesso de peso são diferentes entre os estudos.

Os resultados do presente estudo mostraram que o CS apresenta uma boa capacidade discriminatória (sensibilidade e especificidade) para identificar o excesso de peso em idosos. Achados da literatura mostraram que os escores dos pontos de corte do CS que podem representar um aumento do risco a saúde são divergen- 
tes ${ }^{19}$. Aspectos como a utilização de diferentes métodos (autorreferidos e acelerômetros) ${ }^{19}$ e a utilização de avaliações normativas ${ }^{19}$ dificultam a generalização dos resultados. Contudo, estudo de revisão sistemática ${ }^{19}$ indicou que os riscos a saúde aumentam entre os indivíduos expostos a mais de quatro horas por dia em CS (240 $\mathrm{min} / \mathrm{dia})$. Nesse sentido, os valores encontrados no presente estudo ( $270 \mathrm{~min} /$ dia ou mais para mulheres e $390 \mathrm{~min} / \mathrm{dia}$ ou mais para os homens) estariam condizentes com os escores de risco disponíveis na literatura.

Entre as limitações do estudo, a avaliação do comportamento sedentário realizado por meio de questionário e o fato de ter considerado apenas o tempo gasto sentado como o total de tempo despendido em CS pode ser indicativo de viés de aferição. Por outro lado, como pontos fortes o mesmo incluiu uma amostra representativa de idosos de uma região do país ainda pouco investigada e utilizou instrumentos de avaliação validados previamente.

Conclui-se que o tempo gasto em CS apresenta uma boa capacidade de discriminar o excesso de peso em idosos com diferentes pontos de corte entre os sexos. Recomenda-se a realização de outros estudos com desenhos longitudinais no intuito de ampliar o conhecimento acerca das repercussões do CS no excesso de peso em idosos.

\section{Conflito de interesse}

Os autores declaram não haver conflito de interesses.

\section{Financiamento}

O projeto Monitoramento das Condições de Saúde de Idosos de um Município de Pequeno Porte (MONIDI) recebeu financiamento da Fundação de Amparo a Pesquisa do Estado da Bahia com a concessão de bolsa de Iniciação Científica.

\section{Agradecimentos}

Agradecemos a Secretária Municipal de Saúde do município de Ibicuí, Bahia pelo apoio no desenvolvimento das atividades de pesquisa no município.

\section{Contribuição dos autores}

Jesus AS e Rocha SV, participaram da concepção do estudo, redação do artigo e revisão final do manuscrito.

\section{Referências}

1. Pate RR, O'neill JR, Lobelo F. The evolving definition of "sedentary". Exerc Sport Sci Rev. 2008;36(4):173-8.

2. Koster A, Caserotti P, Patel KV, Matthews CE, Berrigan D, Van Domelen DR et al. Association of Sedentary Time with
Mortality Independent of Moderate to Vigorous Physical Activity. Plos One. 2012;7(6):e37696.

3. Rezende LFM, Rey-López JP, Matsudo VKR, Luiz OC. Sedentary behavior and health outcomes among older adults: a systematic review. BMC Public Health. 2014;14,333.

4. Proper KI, Singh AS, van Mechelen W, Chinapaw MJM. Sedentary behaviors and health outcomes among adults: a systematic review of prospective studies. Am J Prev Med. 2011;40(2):174-82.

5. Tremblay MS, Colley RC, Saunders TJ, Healy GN, Owen N. Physiological and health implications of a sedentary lifestyle. Appl Physiol Nutr Metab. 2010;35(6):725-40.

6. Biswas A, Oh PI, Faulkner GE, Bajaj RR, Silver MA, Mitchell MS, et al. Sedentary time and its association with risk for disease incidence, mortality, and hospitalization in adults: a systematic review and meta-analysis. Ann Intern Med. 2015;162:123-32.

7. Ekelund U, Steene-Johannessen J, Brown WJ, Fagerland MW, Owen N, Powell KE, et al. Does physical activity attenuate, or even eliminate, the detrimental association of sitting time with mortality? A harmonised meta-analysis of data from more than 1 million men and women. Lancet. 2016;388:1302-10.

8. Meneguci J, Sasaki JE, Santos AS, Scatena LM, Damião R. Socio-demographic, clinical and health behavior correlates of sitting time in older adults. BMC Public Health. 2015;15,65.

9. Mielke GI, Silva ICM, Owen N, Hallal PC. Brazilian adults sedentary behaviors by life domain: population based study. Plos One. 2014;9(3):e91614.

10. Jefferis BJ, Parsons, TJ, Sartini, C, Ash S, Lennon LT, Papacosta O, et al. Objectively measured physical activity, sedentary behaviour and all-cause mortality in older men: does volume of activity matter more than pattern of accumulation? 2018. Br J Sports Med. 2017-098733.

11. Donini, LM, Savina C, Gennaro E, De Felice MR, Rosano A, Pandolfo MM, et al. A systematic review of the literature concerning the relationship between obesity and mortality in the elderly. J Nutr Health Aging. 2012;16(1):89-98.

12. Jura M, Kozak LP. Obesity and related consequences to ageing. Age. 2016.38(1):23.

13. Silva VS, Souza I, Petroski EL, Silva DAS. Prevalência e fatores associados ao excesso de peso em idosos brasileiros. Rev Bras Ativ Fís Saúde. 2011;16(4):289-94.

14. Han TS, Tajar A, Lean EJ. Obesity and weight management in the elderly. Br Med Bull. 2011;97:169-96.

15. Gallagher EJ, Le Roith D. Obesity and Diabetes: The Increased Risk of Cancer and Cancer-Related Mortality. Physiol Rev. 2015;95(3):727-48.

16. Giuli C, Papa R, Bevilacqua R, Felici E, Gagliardi C, Marcellini F, et al. Correlates of perceived health related quality of life in obese, overweight and normal weight older adults: an observational study. BMC Public Health. 2014;14:35.

17. De Stefano F, Zambon S, Giacometti L, et al. Obesity, muscular strength, muscle composition and physical performance in an elderly population. J Nutr Health Aging. 2015;19(7):785-91.

18. Chang CI, Huang $\mathrm{KC}$, Chan DC, Wu CH, Lin CC, Hsiuung CA, et al. The impacts of sarcopenia and obesity on physical performance in the elderly. Obes Res Clin Pract. 2015;9(3):256-65.

19. Santos RG, Medeiros JC, Schmitt BD, Meneguci J, Santos DAT, Damião R, et al. Comportamento Sedentário em Idosos: 
uma revisão sistemática. Motricidade. 2015;11(3):171-86.

20. Luiz RR, Magnanini MMF. A lógica na determinação do tamanho da amostra em investigações epidemiológicas. Cad. Saude Colet. 2000;8(2):9-28.

21. Pedreira RBS, Rocha SV, Santos CA, Vasconcelos LRC, Reis MC. Validade de conteúdo do Instrumento de Avaliação da Saúde do Idoso. Einstein. 2016;14(2):158-77.

22. Benedetti TRB, Antunes PC, Rodriguez-Anez CR, Mazo GZ, Petroski EL. Reproducibility and validity of the International Physical Activity Questionnaire (IPAQ) in elderly men. Rev Bras Med Esporte. 2007;13(1):11-6.

23. Lohman TG, Roche AF, Martorell R. Anthropometric Standardization Reference Manual. Champaign, Illinois: Human Kinetics. 1988.

24. Lipschitz DA. Triagem para o estado nutricional nos idosos. Prim Care. 1994;21(1):55-67.

25. Pereira IFS, Spyrides MHC, Andrade LMB. Estado nutricional de idosos no Brasil: uma abordagem multinível. Cad Saúde Pública. 2016;32(5):e00178814.

26. Meneguci J, Santos DAT, Silva RB, Santos RG, Sasaki JE, Tribess S, et al. Comportamento sedentário: conceito, implicações fisiológicas e os procedimentos de avaliação. Motri. 2015;11(1):160-74.

27. Bey L, Hamilton MT. Suppression of skeletal muscle lipoprotein lipase activity during physical inactivity: a molecular reason to maintain daily low-intensity activity. J. Physiol. 2003;55(2):673-82.

28. Bann D, Hire D, Manini T, Cooper R, Botoseneanu A, McDermott MM, et al. Light Intensity physical activity and sedentary behavior in relation to body mass index and grip strength in older adults: cross-sectional findings from the Lifestyle Interventions and Independence for Elders (LIFE) study. Plos One. 2015;10(2):e0116058.

29. Gomez CA, Vicente RG, Pindado M, Vila S, Casajús JA, Pradas AI. Mayor riesgo de obesidad y obesidad central en mujeres post-menopáusicas sedentarias. Nutr Hosp. 2012;27(3):865-70.

30. Monego E, Barbosa AR. Factors associated with daily sitting time in a rural community-dwelling of older adults from southern Brazil. Rev Bras Ativ Fís Saúde. 2014;19(3):371-81.

Recebido: 25/02/2018

Aprovado: 08/10/2018

\section{Como citar este artigo:}

Jesus AS, Rocha SV. Comportamento sedentário como critério discriminador do excesso de peso corporal em idosos. Rev Bras Ati Fis Saúde. 2018;23:e0030. DOI: 10.12820/rbafs.23e0030 